\title{
A NOTE ON HILBERT'S OPERATOR
}

The transformation

H. KOBER

(1) $\mathfrak{S} f=\frac{1}{\pi} P V \int_{-\infty}^{\infty} \frac{f(t)}{t-x} d t=\frac{1}{\pi} \lim _{\epsilon \rightarrow 0} \int_{\epsilon}^{\infty} \frac{d t}{t}\{f(x+t)-f(x-t)\}$

is well known to have the following properties:

LEMma 1.1 When $1<p<\infty$, then $\mathfrak{S} f$ is a continuous (bounded) linear transformation with both domain and range $L_{p}(-\infty, \infty)$, and $\mathfrak{S}^{2} f=-f$.

Lemma 2. ${ }^{2}$ When $f(t) \in L_{1}(-\infty, \infty)$, then $\mathfrak{S} f$ exists for almost all $x$ in $(-\infty, \infty)$, but does not necessarily belong to $L_{1}(a, b)$, where $a, b$ are arbitrarynumbers $(-\infty \leqq a<b \leqq \infty) ;$ however $\left(1+x^{2}\right)^{-1} \mid \mathfrak{S} f^{\prime} q \in L_{1}(-\infty, \infty)$ when $0<q<1$. When $f$ and $\mathfrak{S} f$ belong to $L_{1}(-\infty, \infty)$, then $\mathfrak{S}^{2} f=-f$.

The case $p=1$ appears to present the greatest difficulties. In the present note I shall deal with the set of elements $f(t) \in L_{1}(-\infty, \infty)$ for which $\mathfrak{S} f \in L_{1}(-\infty, \infty)$. In consequence of the lemmas, in this set or in $L_{p}(-\infty, \infty)(1<p<\infty)$, $\mathfrak{g} f$ has no characteristic values other than $\pm i$. We shall start from the sets of characteristic functions and, incidentally, from the class $\mathfrak{S}_{p}$, the theory of which has been developed by E. Hille and J. D. Tamarkin; $\mathfrak{S}_{p}$ is the set of functions $F(z)(z=x+i y)$ which, for $y>0$, are regular and satisfy the inequality

$$
\int_{-\infty}^{\infty}|F(x+i y)|{ }^{p} d x \leqq M^{p} \quad \text { or } \quad|F(z)| \leqq M
$$

for $0<p<\infty$ or $p=\infty$, respectively, where $M$ depends on $F$ and $p$ only. ${ }^{3}$ By $\Omega_{p}$ we denote the corresponding class defined for $y<0$, and by $F(t), G(t)$ the limit-functions ${ }^{3}(y \rightarrow 0 ; x=t)$ of elements $F(z) \in \mathfrak{S}_{p}$, $G(z) \in \Omega_{p}$. By $\mathfrak{S}_{p}^{\prime}$ and $\Omega_{p}^{\prime}$, respectively, we denote the two sets of those limit-functions, and by $\mathfrak{S}_{p}^{\prime}+\mathfrak{R}_{p}^{\prime}$ the smallest linear manifold

Received by the editors August 5, 1941.

${ }^{1}$ M. Riesz, Mathematische Zeitschrift, vol. 27 (1928), pp. 218-244.

${ }^{2}$ E. C. Titchmarsh, Introduction to the Theory of Fourier Integrals, Oxford, 1937, §5.14. E. Hille and J. D. Tamarkin, Fundamenta Mathematicae, vol. 25 (1935), pp. 329-352. Comparing our notation with that of Hille-Tamarkin, we have $\mathfrak{S} f=-\widetilde{f}$.

${ }^{3}$ Loc. cit., $1 \leqq p<\infty$. T. Kawata, Japanese Journal of Mathematics, vol. 13 (1936), pp. 421-430, $0<p<\infty$. The limit-functions exist for almost all $t$ in $(-\infty, \infty)$ and belong to $L_{p}(-\infty, \infty)$. 
containing both $\mathfrak{S}_{p}^{\prime}$ and $\Re_{p}^{\prime}$. Obviously an element $f(t)$ belongs to $\mathfrak{S}_{p}{ }^{\prime}+\mathfrak{R}_{p}^{\prime}$ if and only if it can be represented in the form

$$
f(t)=F(t)+G(t)=F(t)+\bar{F}_{1}(t), \quad F \in \mathfrak{S}_{p}^{\prime}, F_{1} \in \mathfrak{S}_{p}^{\prime}, G \in \mathfrak{\Omega}_{p}^{\prime},
$$

and this representation is unique, except for a constant when $p=\infty$.

Theorem 1 (b), as yet unpublished, is due to H. R. Pitt, Aberdeen, to whom I am greatly indebted.

We obtain the following results:

Lemma 3 . Let $1 \leqq p \leqq \infty$; let the norm of an element $\phi(z)$ belonging to $\mathfrak{S}_{p}$ or $\mathfrak{\Omega}_{p}$ be defined by

$$
|\phi(t)|_{p}=\left\{\int_{-\infty}^{\infty}|\phi(t)|^{p} d t\right\}^{1 / p} \text { or }|\phi(t)|_{p}=\underset{-\infty<t<\infty}{\text { ess.u.b. }}|\phi(t)|
$$

for $1 \leqq p<\infty$ or $p=\infty$, respectively. Then $\mathfrak{S}_{p}$ and $\mathfrak{\Omega}_{p}$ are complete normed linear spaces, that is to say, $(B)$ spaces in the terminology of Banach.

TheOREm 1. Let $f(t) \in L_{1}(-\infty, \infty)$. (a) A necessary condition that $\mathfrak{S} f \in L_{1}(-\infty, \infty)$ is

$$
\int_{-\infty}^{\infty} f(t) d t=0
$$

(b) (Pitt's theorem.) The condition is not sufficient.

THEOREM 2. (a) A necessary and sufficient condition that both $f$ and $\mathfrak{S} f$ belong to $L_{1}(-\infty, \infty)$ is that $f$ belongs to $\mathfrak{S}_{1}^{\prime}+\Omega_{1}^{\prime}$. (b) With domain $\mathfrak{S}_{1}^{\prime}+\mathfrak{\Omega}_{1}^{\prime}, \mathfrak{S} f$ is a linear closed ${ }^{4}$ unbounded transformation in $L_{1}(-\infty, \infty)$.

THEOREM 3. The set $\mathfrak{S}_{1}^{\prime}+\Re_{1}^{\prime}$ is a non-closed subspace of $L_{1}$ and is nowhere dense in $L_{1}$. Its closure is the subset of $L_{1}$ satisfying (5).

We note that, by Lemma 1 and by the argument which will be employed in the proof of Theorem $2(\mathrm{a}), \mathfrak{S}_{p}^{\prime}+\Omega_{p}^{\prime}=L_{p}$ for $1<p<\infty$.

We shall now give the proofs of the above results; some examples will be given at the end of this paper.

Proof of Lemma 3. We need only show that the space $\mathfrak{S}_{p}$ is complete. Let $\left\{F_{n}(z)\right\} \in \mathfrak{S}_{p}, n=1,2, \cdots$, be a sequence satisfying the condition of convergence $\left|F_{n}(t)-F_{m}(t)\right|_{p} \rightarrow 0(m>n \rightarrow \infty)$. Then there exists an element $F(t) \in L_{p}$ such that $\left|F(t)-F_{n}(t)\right|_{p} \rightarrow 0$ as $n \rightarrow \infty$; we have to show that $F(t)$ is the limit-function of an element $\varphi(z) \in \mathfrak{S}_{p}$.

${ }^{4}$ That is to say, $f_{n} \in \mathfrak{S}_{1}^{\prime}+\mathfrak{R}_{1}^{\prime}, g_{n}=\mathfrak{S} f_{n}(n=1,2, \cdots),\left|f-f_{n}\right|_{1} \rightarrow 0$ and $\left|g-g_{n}\right|_{1} \rightarrow 0$ imply $g=\mathfrak{g} f$. 
By a result due to Hille and Tamarkin, ${ }^{5} F_{n}(z)$ is represented by its "proper Poisson integral"

Let

$$
\begin{aligned}
F_{n}(z)=\frac{1}{\pi} \int_{-\infty}^{\infty} \frac{y F_{n}(t) d t}{(t-x)^{2}+y^{2}}=\frac{1}{\pi} \int_{-\infty}^{\infty} \frac{y F_{n}(t+x)}{t^{2}+y^{2}} d t \\
z=x+i y ; y \rightarrow 0 .
\end{aligned}
$$

$$
\varphi(z)=\frac{1}{\pi} \int_{-\infty}^{\infty} \frac{y F(t+x)}{t^{2}+y^{2}} d t .
$$

For $y \geqq \epsilon>0$, by Hölder's theorem, we have uniformly

$$
\left|\varphi(z)-F_{n}(z)\right| \leqq \pi^{-1} \epsilon^{-1 / p}\left|\left(t^{2}+1\right)^{-1}\right|_{p^{\prime}}\left|F(t)-F_{n}(t)\right|_{p} \rightarrow 0
$$

as $n \rightarrow \infty$, where $1 / p+1 / p^{\prime}=1$. Hence $\varphi(z)$ is a regular function for $y>0$, and it is obviously bounded when $p=\infty$.

Now let $1 \leqq p<\infty$. By a well known convexity theorem,

$$
\begin{aligned}
\int_{-\infty}^{\infty}|\varphi(x+i y)|^{p} d x & \leqq \frac{1}{\pi} \int_{-\infty}^{\infty} \frac{y d t}{t^{2}+y^{2}} \int_{-\infty}^{\infty}|F(t+x)|^{p} d x \\
& =\int_{-\infty}^{\infty}|F(x)|^{p} d x .
\end{aligned}
$$

Thus $\varphi(z) \in \mathfrak{S}_{p}$. By the same argument and by Fatou's theorem,

$$
\begin{aligned}
\left|\varphi(t)-F_{n}(t)\right|_{p} & \leqq \liminf _{y \rightarrow 0}\left\{\int_{-\infty}^{\infty}\left|\varphi(z)-F_{n}(z)\right|^{p} d x\right\}^{1 / p} \\
& \leqq\left|F(t)-F_{n}(t)\right|_{p} \rightarrow 0 .
\end{aligned}
$$

By (8), the result holds for $p=\infty$. Therefore $F(t) \equiv \varphi(t)$, which completes the proof.

To prove Theorem 2(a) we require a result which we deduce from theorems by Hille and Tamarkin:

Lemma 4. A necessary and sufficient condition that $f(t)$ belongs to $L_{p}(-\infty, \infty)(1 \leqq p<\infty)$ and that $\mathfrak{S} f=$ if or $\mathfrak{S} f=-$ if is that $f(t)$ belongs to $\mathfrak{S}_{p}^{\prime}$ or $\mathfrak{\Omega}_{p}^{\prime}$, respectively.

Let $f(t) \in \mathfrak{S}_{p}^{\prime}$ and $f(t)=\varphi(t)+i \psi(t)$. Since $f(t)$ is the limit-function $(y \rightarrow 0, x=t)$ of an element $F(z) \in \mathfrak{S}_{p}$, and since $F(z)$ is represented by its proper Poisson integral, we have ${ }^{6} \psi(x)=-\mathfrak{S} \varphi$ and, by Lemmas 1

${ }^{5}$ Loc. cit., Theorem 2.1 (ii), $1 \leqq p<\infty$. The result holds for $p=\infty$.

${ }^{6}$ Hille and Tamarkin, loc. cit., Theorem 3.1. For $p=2$, the lemma is an easy consequence of Theorem 95, Titchmarsh, loc. cit. 
and 2, $\varphi(x)=\mathfrak{S} \psi$; therefore $\mathfrak{S} f=\mathfrak{S}(\varphi+i \psi)=-\psi+i \varphi=i f$. Conversely, let $f(t) \in L_{p}$ and $\mathfrak{S} f=i f$. For $y>0$, the function

$$
F(z)=\frac{1}{2 \pi i} \int_{-\infty}^{\infty} \frac{f(t)}{t-z} d t
$$

is regular and representable by its proper Poisson integral, and its limit function is $(1 / 2)\{f(x)-i \mathfrak{S} f\}=(1 / 2)(f(x)+f(x))=f(x) .^{6}$ Hence $F(z) \in \mathfrak{S}_{p}$, and so $f(t) \in \mathfrak{S}_{p}^{\prime}$, which proves the lemma.

Proof of Theorem 2(a). Let $f \in L_{1}$ and $\mathfrak{S} f \in L_{1}$, then the functions

$$
F=(1 / 2)(f-i \mathfrak{g} f), \quad G=(1 / 2)(f+i \mathfrak{g} f)
$$

belong to $L_{1}$; by Lemma $2, \mathfrak{S}^{2} f=-f$, and so $\mathfrak{S} F=i F, \mathfrak{S} G=-i G$. By Lemma 4 , we have $F \in \mathfrak{S}_{1}^{\prime}, G \in \Omega_{1}^{\prime}$, and so $f=F+G \in \mathfrak{S}_{1}^{\prime}+\Omega_{1}^{\prime}$. 4 ,

Conversely, let $f \in \mathfrak{S}_{1}^{\prime}+\Omega_{1}^{\prime}$. Then, by (3) with $p=1$, and by Lemma

$$
\mathfrak{S} f=\mathfrak{S} F+\mathfrak{S} G=i(F-G) \in L_{1}(-\infty, \infty) \text {, }
$$

which proves Theorem 2(a). Part (b) will be proved after Theorem 3 .

To prove Theorem 1 , we need a further result due to Hille and Tamarkin. ${ }^{7}$

Lemma 5. Let $1 \leqq p<\infty$, Let $\phi(t)$ belong to $L_{p}$ and possess a Fourier transform $\psi(x)$,

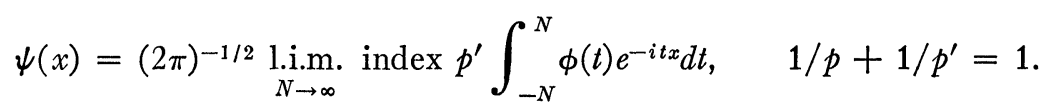

Then $\phi(t) \in \mathfrak{S}_{p}^{\prime}$ or $\mathfrak{\Omega}_{p}^{\prime}$ if and only if $\psi(x)$ vanishes in $(-\infty, 0)$ or in $(0, \infty)$, respectively.

For completeness we add the following result:

Lemma $5^{\prime}$. Let $2<p<\infty$ and let $\phi(t)$ belong to $L_{p}$ and have no Fourier transform in $L_{p^{\prime}}$. Then $\phi(t) \in \mathfrak{S}_{p}^{\prime}$ or $\Omega_{p}^{\prime}$ if and only if there is a sequence $\left\{\phi_{n}(t)\right\}$ belonging to $\mathfrak{S}_{p}^{\prime}$ or $\mathfrak{\Omega}_{p}^{\prime}$ and satisfying the hypotheses of Lemma 5 and such that $\left|\phi(t)-\phi_{n}(t)\right|_{p} \rightarrow 0$ as $n \rightarrow \infty .^{8}$

${ }^{7}$ Loc. cit., Lemma 4.2, and Annals of Mathematics, (2), vol. 34 (1933), pp. 606614 , Theorem 3.

${ }^{8}$ The proof is similar to that given for the generalization of a theorem due to Paley-Wiener; H. Kober, Quarterly Journal of Mathematics, vol. 11 (1940), pp. 66-

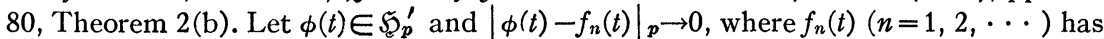
a Fourier transform in $L_{p}{ }^{\prime}$; then the functions $\phi_{n}(t)=(1 / 2)\left(f_{n}-i \mathfrak{S}_{\mathfrak{g}} f_{n}\right)$ have the desired properties. The converse is proved by Lemma 3. 
By Theorem 2 and Lemma $5, f(t)$ can be represented as the sum of two functions $F(t), G(t)$ belonging to $L_{1}$ and such that their Fourier transforms $\varphi(x), \psi(x)$ vanish for $x<0$ or $x>0$, respectively. By continuity, they also vanish at $x=0$; so does the Fourier transform of $f(t)$, which gives (5). For the Fourier transform of an element $F(t) \in L_{1}$ is continuous in $(-\infty, \infty)$.

To prove Theorem $1(\mathrm{~b})$, take $f_{1}(t)=t^{-1} \log ^{-2} t$ and $f_{2}=2 / \log 2$ in $(0,1 / 2), f_{1}(t)=f_{2}(t)=0$ otherwise. Let $f(t)=f_{1}(t)-f_{2}(t)$, then obviously $f(t)$ belongs to $L_{1}(-\infty, \infty)$ and satisfies (5). But $\mathfrak{g} f$ does not belong to $L_{1}(-\infty, \infty)$, since

$$
\begin{gathered}
\int_{-1 / 2}^{0}\left|\mathfrak{S} f_{1}\right| d x=\infty, \\
\int_{-1 / 2}^{0}\left|\mathfrak{S} f_{2}\right| d x=\frac{2}{\pi \log 2} \int_{-1 / 2}^{0}|\log | 1-\frac{1}{2 x}|| d x<\infty .
\end{gathered}
$$

For, in $(0,1 / 2)$, we have

$$
\begin{aligned}
\mathfrak{S}\left[f_{1} ;-x\right] & =\frac{1}{\pi} \int_{0}^{1 / 2} \frac{\log ^{-2} t d t}{(x+t) t} \geqq \frac{1}{\pi} \int_{0}^{x} \frac{\log ^{-2} t d t}{(x+t) t} \\
& \geqq \frac{1}{\pi} \int_{0}^{x} \frac{\log ^{-2} t d t}{2 x t}=\frac{1}{2 \pi x|\log x|}
\end{aligned}
$$

hence $\mathfrak{S} f_{1}$ does not belong to $L_{1}(-1 / 2,0)$, which proves the theorem.

Proof of Theorem 3. Let $E$ be the subset of $L_{1}$ satisfying (5). By Theorems 1 and 2, $\mathfrak{S}_{1}^{\prime}+\Omega_{1}^{\prime}$ is a subset of $E$ and different from $E$. It is easy to see that $E$ is closed in $L_{1}$. We are left to show that $E$ is the closure of $\mathfrak{S}_{1}^{\prime}+\Omega_{1}^{\prime}$.

Let $f(t)$ be a step-function belonging to $E$. Denoting by $e(t)$ the step-function which is equal to 1 in $(0,1)$ and to zero otherwise, we can represent $f(t)$ by a finite sum $\sum a_{n} \mathrm{e}\left(t / b_{n}\right)\left(b_{n} \lessgtr 0, a_{n}\right.$ complex). By (5), $\sum a_{n}\left|b_{n}\right|=0$, and so

$$
\pi \mathfrak{S} f=\sum a_{n}\left(\log \left|1-\frac{b_{n}}{x}\right|\right) \operatorname{sgn} b_{n}=O\left(x^{-2}\right), \quad x \rightarrow \pm \infty .
$$

Hence $\mathfrak{S} f \in L_{1}, f \in \mathfrak{S}_{1}^{\prime}+\Omega_{1}^{\prime}$. We can now approximate to any $f(t) \in E$ by a sequence $\left\{f_{n}(t)\right\}(n=1,2, \cdots)$ of step-functions belonging to $\mathfrak{S}_{1}^{\prime}+\Omega_{1}^{\prime}$. Let $f(t)$ satisfy (5), and let $\left\{g_{n}(t)\right\}$ be a sequence of stepfunctions such that $\left|f(t)-g_{n}(t)\right|_{1 \rightarrow 0}$ as $n \rightarrow \infty$. Take

$$
f_{n}(t)=g_{n}(t)-\mathrm{e}(t) \int_{-\infty}^{\infty} g_{n}(\xi) d \xi, \quad n=1,2, \ldots .
$$


Then $f_{n}(t)$ is a step-function, $f_{n}(t)$ satisfies (5); therefore $f_{n} \in \mathfrak{S}_{1}^{\prime}+\Omega_{1}^{\prime}$. Finally, by (5), we have

$$
\begin{aligned}
\left|f-f_{n}\right|_{1} & =\left|f-g_{n}-e \int_{-\infty}^{\infty}\left\{f(\xi)-g_{n}(\xi)\right\} d \xi\right| 1 \\
& \leqq\left|f-g_{n}\right|_{1}+\left|\int_{-\infty}^{\infty}\left\{f(\xi)-g_{n}(\xi)\right\} d \xi\right| \leqq 2\left|f-g_{n}\right|_{1},
\end{aligned}
$$

which tends to zero as $n \rightarrow \infty$. Thus $E$ is the closure of $\mathfrak{S}_{1}^{\prime} \dot{+} \Re_{1}^{\prime}$.

The set $E$ is nowhere dense in $L_{1}$. For when $f \in L_{1}$, then, given $\epsilon>0$, any element $g(t)=f(t)+\delta \mathfrak{e}(t) \quad(0<|\delta|<\epsilon)$ belongs to the sphere $|g-f|_{1}<\epsilon$, while $g$ does not belong to $E$; when $f$ belongs to $L_{1}$ but not to $E$, then no element $g$ of the sphere $|g-f|_{1}<\left|\int f(t) d t\right|$ belongs to $E$. Thus we have proved the theorem.

Proof of Theorem 2(b). In the domain $\mathfrak{S}_{1}^{\prime}+\Re_{1}^{\prime}$, by Lemma 2, we have $i \mathfrak{g}(i \mathfrak{g} f)=f$; hence $i \mathfrak{g} f$ is involutory. By Lemma 3 , both $\mathfrak{S}_{1}^{\prime}$ and $\Re_{1}^{\prime}$ are closed spaces in $L_{1}$. Therefore $i \mathfrak{g} f$, and therefore $\mathfrak{S} f$, is closed; for a linear involutory transformation in a $(B)$ space is closed if and only if the spaces of the characteristic functions are closed. ${ }^{9} \mathrm{By}$ Theorem $3, \mathfrak{S}_{1}^{\prime} \dot{+} \Re_{1}^{\prime}$ is not closed. Therefore $\mathfrak{S} f$ is not bounded in this domain; for a linear closed transformation in a $(B)$ space is continuous if and only if its domain is closed. ${ }^{10}$ Thus we have proved the theorem.

The following are examples for the case $f \in L_{1}(-\infty, \infty), \mathfrak{S} f$ $\in L_{1}(-\infty, \infty)$. We may start from Lemma $4,{ }^{11}$ but it is easier to make use of Theorem 2 .

(1) Let $T_{1}(z)$ or $T_{2}(z)$ be polynomials of degree $\alpha>0$ or $\beta>0$ and such that they have no zeros for $y \geqq 0$ or $y \leqq 0$, respectively; let $a, b$ be any numbers such that $-\infty<a<-1 / \alpha,-\infty<b<-1 / \beta$. Then, on a suitable Riemann surface, any branch of $\left\{T_{1}(z)\right\}^{a}(y>0)$ or $\left\{T_{2}(z)\right\}^{b}$ $(y<0)$ belongs to $\mathfrak{S}_{1}$ or $\Re_{1}$, respectively. When $f(t)=\left\{T_{1}(t)\right\}^{a}$ $+\left\{T_{2}(t)\right\}^{b}$, by Lemma 4 , we have $\mathfrak{g} f=i\left\{T_{1}(x)\right\}^{a}-i\left\{T_{2}(x)\right\}^{b} \in L_{1}$.

(2) Let $\varphi_{1}(z)=(1-\cos \alpha z) z^{-2}, \varphi_{2}(z)=\{\sin \alpha z-2 \sin (\alpha z / 2)\} z^{-2}, \alpha>0$, and let $f(t)=A \varphi_{1}(t) e^{i \alpha t}+B \varphi_{2}(t) e^{-i \alpha t}$; then $\mathfrak{S}_{e} f=i A \varphi_{1}(x) e^{i \alpha x}-i B \varphi_{2}(x)^{-i \alpha x}$, and $f \in L_{1}, \mathfrak{S} f \in L_{1}$. It can be shown that this result holds when $\varphi_{j}(z)(j=1,2)$ are integral functions such that $\varphi_{j}(t) \in L_{1}$ and that, for any $\epsilon>0,\left|\varphi_{j}(z)\right|<K_{\epsilon} \exp \{(\alpha+\epsilon)|z|\}$; in this way we can construct

${ }^{9} \mathrm{H}$. Kober, Proceedings of the London Mathematical Society, (2), vol. 44 (1938), pp. 453-465, Theorem $6^{\prime}(a)$.

${ }^{10} \mathrm{~S}$. Banach, Théorie des Opérations Linéaires, Warsaw, 1932, p. 41, Theorem 7. Probably the converse is well known.

${ }^{11}$ Or from Theorem 3.1, Hille and Tamarkin, loc. cit. 
all integral functions $f(z)$ satisfying the conditions $f(t) \in L_{1}, \mathfrak{S} f \in L_{1}$, $|f(z)|<K_{f, \epsilon} \exp \{(2 \alpha+\epsilon)|z|\}$. The proof is based upon a result due to Plancherel and Pólya. ${ }^{12}$

The University,

Edgbaston, Birmingham, England

${ }^{32}$ Commentarii Mathematici Helvetici, vol. 10 (1937-1938), pp. 110-163, §27.

\title{
THE BEHAVIOR OF CERTAIN STIELTJES CONTINUED FRACTIONS NEAR THE SINGULAR LINE
}

\author{
H. S. WALL
}

1. Introduction. We consider here continued fractions of the form ${ }^{1}$

(1.1) $f(z)=\frac{g_{0}}{1}+\frac{g_{1} z}{1}+\frac{\left(1-g_{1}\right) g_{2} z}{1}+\frac{\left(1-g_{2}\right) g_{3} z}{1}+\cdots$,

in which $g_{0} \geqq 0,0 \leqq g_{n} \leqq 1,(n=1,2,3, \cdots)$, it being agreed that the continued fraction shall terminate in case some partial numerator vanishes identically. There exists a monotone non-decreasing function $\phi(u), 0 \leqq u \leqq 1$, such that

$$
f(z)=\int_{0}^{1} \frac{d \phi(u)}{1+z u} ;
$$

and, conversely, every integral of this form is representable by such a continued fraction. Put $M(f)=1$.u.b.|z|<1 $|f(z)|$. Then $M(f) \leqq 1$ if and only if the continued fraction can be written in the form

$$
f(z)=\frac{h_{1}}{1}+\frac{\left(1-h_{1}\right) h_{2} z}{1}+\frac{\left(1-h_{2}\right) h_{3} z}{1}+\cdots,
$$

in which $0 \leqq h_{n} \leqq 1,(n=1,2,3, \cdots)$. These functions are analytic in the interior of the $z$-plane cut along the real axis from $z=-1$ to $z=-\infty$.

The principal object of this paper is to prove the following theorem:

THEOREM 1.1. If $0<h_{n}<1,(n=1,2,3, \cdots)$, and $h_{n} \rightarrow 1 / 2$ in such $a$ way that the series $\sum\left|h_{n}-1 / 2\right|$ converges, then the function $f(z)$ given

Presented to the Society, October 25, 1941; received by the editors August 14, 1941.

${ }^{1} \mathrm{H}$. S. Wall, Continued fractions and totally monotone sequences, Transactions of this Society, vol. 48 (1940), pp. 165-184. 